\title{
Neutrophil-Lymphocyte Ratio as an Initial Screening Biomarker for Differential Diagnosis of Cushing's Syndrome from Nonfunctional Adenoma in Patients with an Adrenal Mass
}

\author{
Wei Wang ${ }^{D},{ }^{1}$ Jianing Wang, ${ }^{1,2}$ Cheng Shen, ${ }^{3}$ Sainan Zhu, ${ }^{4}$ Ying Gao ${ }^{\circ},{ }^{1}$ \\ and Junqing Zhang ${ }^{1}$ \\ ${ }^{1}$ Department of Endocrinology, Peking University First Hospital, No. 8 Xishiku Street, Beijing 100034, China \\ ${ }^{2}$ Department of Endocrinology, Beijing Longfu Hospital, No. 18 Art Museum East Street, Beijing 100010, China \\ ${ }^{3}$ Department of Urology, Peking University First Hospital, No. 8 Xishiku Street, Beijing 100034, China \\ ${ }^{4}$ Department of Medical Statistics, Peking University First Hospital, No. 8 Xishiku Street, Beijing 100034, China
}

Correspondence should be addressed to Ying Gao; bjgaoying@yahoo.com

Wei Wang and Jianing Wang contributed equally to this work.

Received 15 November 2020; Revised 25 December 2020; Accepted 1 February 2021; Published 15 February 2021

Academic Editor: Takashi Yazawa

Copyright ( 2021 Wei Wang et al. This is an open access article distributed under the Creative Commons Attribution License, which permits unrestricted use, distribution, and reproduction in any medium, provided the original work is properly cited.

\begin{abstract}
Objective. Assessing excess adrenal hormones is important in patients with adrenal mass. Current screening tests for excess cortisol hormones are complex, so it cannot be done sometimes due to the limited medical resources. The aim of the study was to evaluate whether the neutrophil-lymphocyte ratio (NLR) can be used as an initial screening biomarker for Cushing's syndrome (CS) in patients with an adrenal mass. Methods. This retrospective study included a total of 185 patients with CS and 185 patients with nonfunctional adrenal adenoma (matched $1: 1$ by sex, body mass index, and discharge date). The NLR was compared between the two groups. The association between NLR and serum and urinary cortisol concentrations was analyzed, and an NLR cut-off value for CS screening was calculated. Results. NLR $(3.38(2.33,5.45)$ vs. $2.13(1.74,3.00), P<0.001)$ was significantly higher in the CS group than in the nonfunctional adenoma group. In CS patients, the NLR was positively associated with serum cortisol concentrations at $8 \mathrm{am}$, with 24-hour urine free cortisol and with serum cortisol after a $1 \mathrm{mg}$ dexamethasone suppression test $(P<0.001$ each). An NLR cut-off of 2.2 had a sensitivity of $80.0 \%$ and a specificity of $54.05 \%$. The weighted Youden index for the NLR was similar to that of the 24-hour urine free cortisol and late-night serum cortisol tests, which are recommended initial tests for CS diagnosis. Conclusion. The NLR may be useful for initial screening for CS among patients with an adrenal mass as an easy and convenient marker.
\end{abstract}

\section{Introduction}

The frequency of detection of adrenal masses has increased in recent years after the introduction of high-resolution crosssectional imaging. Previous studies report a frequency of around $3 \%$ in subjects aged 50 years, increasing to about $10 \%$ in elderly individuals [1-5]. The European Society of Endocrinology and European Network for the Study of Adrenal Tumors guidelines on adrenal incidentaloma recommend that adrenal hormone concentrations in patients with adrenal incidentaloma are measured to identify those with adrenal hormone excess [6]. About $12 \%$ of patients with adrenal incidentaloma have Cushing's syndrome (CS), a condition characterized by constitutive cortisol-secreting adenomas and associated with severe morbidity and elevated mortality. Despite available treatments for comorbidities, patients with active CS continue to have a standardized mortality rate 1.7 - to 4.8 -fold greater than the general population [7-11]. Early diagnosis of CS can improve patient prognosis.

The Chinese experts' consensus for Cushing's syndrome (2011) recommends two steps for CS diagnosis. Initial screening includes measurement of 24-hour urine free cortisol 
(UFC), late-night saliva cortisol, and serum cortisol rhythms. If the results in initial screening are abnormal, further diagnostic indicators include serum cortisol levels greater than $1.8 \mu \mathrm{g} / \mathrm{dL}$ after overnight $1 \mathrm{mg}$ dexamethasone or low-dose dexamethasone ( $2 \mathrm{mg}$ /day for 48 hours) suppression tests (LDDST), [12]. Since tests for serum cortisol and UFC are available only in some large hospitals and the saliva cortisol test can be performed by only a few hospitals, special endocrinology tests may not always be available in underdeveloped regions of China. Initial screening and further diagnostic tests for CS cannot be performed due to stringent requirements regarding detection methods and sample collection; this is a problem for primary care clinics or underdeveloped regions where medical resources are scarce. Therefore, more easily measurable biomarkers are needed to screen for CS.

Previous studies show that elevated white blood cell (WBC) counts in Cushing's disease are associated with hypercortisolism; this is because glucocorticoid receptors expressed by WBCs play a role in cell adhesion and WBC recruitment from the bone marrow [13]. Additionally, there is a significant positive correlation between decreased UFC levels and decreased WBC counts after surgery; similar changes in WBC counts are observed in pediatric CS patients [14]. Furthermore, neutrophil counts are significantly higher, and lymphocyte counts are significantly lower, in CS patients than in controls. The neutrophil-lymphocyte ratio (NLR) is an index calculated by dividing the neutrophil count by the lymphocyte count, which may be associated with cortisol levels. Studies show that the NLR is associated with disease activity and prognosis in patients with tumors, cardiovascular disease, and autoimmune disease [15-19]. However, little is known about the NLR in patients with CS. The NLR is easier to measure during routine blood testing than during current initial tests for CS. The aim of the present study was to investigate whether the NLR is an appropriate screening biomarker for CS.

\section{Materials and Methods}

2.1. Study Population. The electronic medical record system of Peking University First Hospital was searched to identify patients with an adrenal mass confirmed by CT scanning from January 2014 to March 2019. Adrenal CS was diagnosed as serum cortisol levels greater than $1.8 \mu \mathrm{g} / \mathrm{dL}$ after LDDST, according to the criteria of the Endocrine Society clinical practice guidelines [20]. Nonfunctional adrenal adenoma was diagnosed by functional assessment, excluding patients with CS [20], primary aldosteronism [21], pheochromocytoma [22], and infiltrative diseases such as adrenal lymphoma and metastatic carcinoma. Patients were included if they had been hospitalized for an adrenal mass, their medical data were complete, and they were discharged with a diagnosis of adrenal CS or nonfunctional adrenal adenoma. Patients were excluded if they had an active infection, a disease of the hematologic system, cardiovascular disease, autoimmune disease, or a nonadrenal tumor. Pregnant women were also excluded, as were those with factors that could affect neutrophil or lymphocyte counts. The diagnostic criteria for an active infection were symptoms or signs of infection, or detection of infection in samples of urine, stool, or other body fluids, or use of antibiotics at the time.

2.2. Ethics. This study conformed to the principles of the Declaration of Helsinki. The study protocol was approved by the Ethics Committee of Peking University First Hospital (approval number 2019-300).

2.3. Study Design. This retrospective analysis included patients treated at a single research hospital. Factors recorded included demographic characteristics (e.g., age, sex, height, weight, and body mass index (BMI)), history of illness, diagnosis at discharge, results of physical examination (e.g., blood pressure and heart rate on the first day of hospitalization), routine blood tests, and measurements of plasma glucose, lipid, electrolyte, and C-reactive protein concentrations (CRP). Other factors included serum cortisol and ACTH concentration rhythms, 24-hour UFC, 8 am serum cortisol after $1 \mathrm{mg}$ DST, the results of adrenal CT scans, and posoperative histopathology. All patients were admitted to the hospital for a period of 48 hours or longer before midnight cortisol sampling to avoid false-positive responses due to the stress of hospitalization. The patient was in sleeping before midnight sampling; the blood sample must be drawn within 5-10 minutes of waking [20]. Patients with CS were divided into those with overt CS and those with adrenal incidentaloma with mild autonomous cortisol excess (AIMCE), depending on whether they presented with or without specific signs and symptoms of CS [23, 24]. AIMCE was defined as autonomous cortisol secretion in the absence of the classical clinical features of Cushing's syndrome in the adrenal incidentaloma [6].

Histopathology of ACTH-independent CS usually includes adrenocortical adenoma, adrenocortical hyperplasia, oncocytic adenoma, and adrenocortical carcinoma. NLR was calculated by dividing the neutrophil count $\left(\times 10^{9} / \mathrm{L}\right)$ by the lymphocyte count $\left(\times 10^{9} / \mathrm{L}\right)$. The weighted Youden index was calculated as $2 \times[\omega \times$ sensitivity $+(1-\omega) \times$ specificity $]$ -1 , where $\omega$ is the weighted coefficient of sensitivity. For the screening test, sensitivity carried more weight, so $\omega$ was set at 0.7 .

2.4. Statistical Analysis. A normality test identified age, BMI, blood pressure, plasma potassium, plasma lipid, neutrophil count, and lymphocyte count as being normally distributed, whereas the NLR, serum cortisol and ACTH concentrations, CRP levels, the longest diameter of adrenal nodules, HbAlc, and fasting plasma glucose were nonnormally distributed. Normally distributed parameters were expressed as the mean \pm standard deviation and were analyzed using Student's $t$ tests. Nonnormally distributed parameters were expressed as the median (interquartile range) and analyzed using the Mann-Whitney $U$ test. The NLR of multiple subgroups was compared using the Kruskal-Wallis test. Categorical data were reported as percentages and compared using the Chi-square test. The correlation between NLR and serum and urinary cortisol levels was determined by Spearman's correlation analysis. The cut-off NLR value for CS was determined by receiver operating characteristic (ROC) 
curves analysis. Areas under the ROC curves for the NLR, neutrophil count, and lymphocyte count were compared using MedCalc software. The sensitivity and specificity of the different tests were compared using McNemar's test. A $P$ value $<0.05$ was considered statistically significant.

\section{Results}

3.1. Characteristics of the CS and Nonfunctional Adrenal Mass Groups. A review of medical records identified 185 patients who had been diagnosed with adrenal CS during the study period. These patients were matched $1: 1$ by sex, BMI $\left( \pm 0.5 \mathrm{~kg} / \mathrm{m}^{2}\right)$, and discharge date, with 185 patients diagnosed with a nonfunctional adrenal mass. In addition to serum cortisol and ACTH concentrations, there were significant between-group differences in UFC, age, systolic and diastolic blood pressure, total cholesterol, plasma potassium concentrations, CRP levels, and adrenal nodule size (Table 1).

3.2. The NLR in the CS and Nonfunctional Adrenal Mass Groups. WBC counts $\left(7.25 \pm 2.16\right.$ vs. $6.35 \pm 1.63\left(\times 10^{9} / \mathrm{L}\right)$, $P<0.001)$ and neutrophil counts $(5.17 \pm 2.11$ vs. $3.96 \pm$ $\left.1.34\left(\times 10^{9} / \mathrm{L}\right), \quad P<0.001\right)$ were significantly higher, and lymphocyte counts $\left(1.45 \pm 0.53\right.$ vs. $1.75 \pm 0.53\left(\times 10^{9} / \mathrm{L}\right)$, $P<0.001)$ were significantly lower in the CS group than in patients with a nonfunctional adrenal mass. The NLR (3.38 $(2.33,5.45)$ vs. $2.13(1.74,3.00), P<0.001)$ was significantly higher in the CS group than in the nonfunctional adrenal mass group (Figure 1).

3.3. Correlation between Serum and Urinary Cortisol Levels and the NLR in the CS Group. After adjusting for BMI, the NLR in the CS group correlated significantly with $8 \mathrm{am}$ serum cortisol $(r=0.574, \quad P<0.001), 24$-hour UFC $(r=0.582, P<0.001)$, and 8 am serum cortisol levels after the $1 \mathrm{mg} \operatorname{DST}(r=0.603, P<0.001)$ (Figures $2(\mathrm{a})-2(\mathrm{c}))$. These positive correlations were also observed after removing an outlier ( $r$ was 0.567 (NLR vs. 8 am serum cortisol concentration), 0.575 (NLR vs. 24-hour UFC), and 0.596 (NLR vs. 8 am serum cortisol after the $1 \mathrm{mg}$ DST); $P<0.001$ for each; data not shown).

3.4. Comparison of the NLR in Different CS Subgroups. A comparison of the NLR between the overt CS and AIMCE subgroups showed that the NLR was significantly higher in patients with overt CS than in those with AIMCE (Table 2). Next, the NLR was compared between different histopathologic subgroups. Histopathologic analysis of the 133 patients with ACTH-independent CS who underwent surgery resulted in a diagnosis of adrenocortical adenoma in 82 patients, adrenocortical hyperplasia in 11 , oncocytic adenoma in 35, and adrenocortical carcinoma in five. The NLR in these subgroups was $4.04(2.80,6.15), 3.40(2.73,5.81)$, $4.18(2.18,6.21)$, and $3.43(2.74,5.23)$, respectively, and did not differ significantly among them.

3.5. Area under the ROC Curves for the NLR for Screening Patients with an Adrenal Mass for CS and Calculating the NLR Cut-Off Value. The 370 samples from the two groups were combined to assess the cut-off value for the NLR when screening for CS. ROC analysis showed that the area under the curve for the NLR was $0.729(P<0.001)$. The area under the ROC curve for NLR was significantly higher than that for the neutrophil count $(P=0.0043)$ and that for the lymphocyte count $(P=0.0067)$ (Figure 3$)$. A cut-off NLR value of 2.2 for CS diagnosis yielded a sensitivity of $80.0 \%$ and a specificity of $54.05 \%$.

3.6. Comparison of the NLR with Other Markers for Screening $C S$. Finally, we compared the sensitivity and specificity of the NLR with those of 24-hour UFC (cut-off, upper limit of normal range), late-night serum cortisol (cut-off, $1.8 \mu \mathrm{g} / \mathrm{dL}$ ), and $1 \mathrm{mg}$ overnight DST (cut-off, $1.8 \mu \mathrm{g} / \mathrm{dL}$ ). The sensitivity and specificity of the NLR was between those of the 24hour UFC and those of the late-night serum cortisol tests. The weighted Youden index of these biomarkers was calculated to show diagnostic accuracy (Table 3). The diagnostic accuracy of the NLR was similar to that of the 24-hour UFC and the late-night serum cortisol tests.

\section{Discussion}

Previous studies demonstrate that elevated WBC counts in $\mathrm{CS}$ are associated with hypercortisolism. Moreover, lymphocyte counts are significantly lower and neutrophil counts significantly higher, in CS patients than in controls. After surgery, WBC counts decrease with the decline in serum cortisol $[13,14]$. We also observed these changes in the CS patients in our study. Additionally, many studies report that the mechanism of changes in lymphocyte counts and neutrophil counts involves an increase in cortisol concentration. The bone marrow of CS patients release high numbers of neutrophils. The marginated pool of neutrophils is mobilized into the circulating pool, due to elevation of cortisol levels. Meanwhile, cortisol inhibits apoptosis of human neutrophils and increases the half-life of circulating neutrophils. At the same time, hypercortisolemia reduces lymphocyte numbers via an immunosuppressive mechanism [13, 14, 25-28]. However, tests based solely on the results of data obtained from routine blood tests are insufficient for screening patients with suspected CS. Because the NLR amplified the difference than solely blood cell counts, it can distinguish between the two groups much better than blood cell counts. In our study, we found that the NLR was significantly higher in patients with CS than in those with a nonfunctional adrenal mass, and subgroup analysis revealed that the NLR was significantly higher in patients with overt CS than in those with AIMCE. The NLR was positively associated with cortisol levels in CS patients. These findings suggest that the NLR may reflect, at least in part, cortisol concentrations and may be a potential biomarker in initial CS tests.

Previous studies report that the NLR is valuable for predicting disease activity and prognosis in patients with tumors, cardiovascular disease, and autoimmune disease [15-19]. A higher NLR is associated with autoimmune disease activity and with poorer prognosis in patients with tumors and cardiovascular disease. To date, no published studies have assessed the relationships between the NLR and a diagnosis of CS. Here, we found that the NLR in 
TABLE 1: Baseline characteristics of the CS and nonfunctional adrenal mass groups.

\begin{tabular}{|c|c|c|c|}
\hline & CS $(n=185)$ & Nonfunctional $(n=185)$ & $P$ value \\
\hline Male (\%) & $61(32.97 \%)$ & $61(32.97 \%)$ & - \\
\hline Age (years) & $47.68 \pm 13.82$ & $54.71 \pm 10.89$ & $<0.001$ \\
\hline BMI $\left(\mathrm{kg} / \mathrm{m}^{2}\right)$ & $25.98 \pm 3.75$ & $26.00 \pm 3.66$ & 0.947 \\
\hline Hypertension (\%) & $151(81.62 \%)$ & $124(67.03 \%)$ & 0.001 \\
\hline Diabetes (\%) & $63(34.05 \%)$ & $51(27.57 \%)$ & 0.177 \\
\hline SBP $(\mathrm{mmHg})$ & $144.65 \pm 18.84$ & $138.77 \pm 18.30$ & 0.002 \\
\hline $\mathrm{DBP}(\mathrm{mmHg})$ & $88.17 \pm 14.60$ & $80.44 \pm 12.79$ & $<0.001$ \\
\hline HbAlc (\%) & $6.0(5.7,6.8)$ & $6.0(5.7,6.6)$ & 0.573 \\
\hline $\mathrm{FPG}(\mathrm{mmol} / \mathrm{L})$ & $5.31(4.72,6.34)$ & $5.47(5.04,6.30)$ & 0.141 \\
\hline Plasma $\mathrm{K}+(\mathrm{mmol} / \mathrm{L})$ & $3.55 \pm 0.44$ & $3.70 \pm 0.33$ & $<0.001$ \\
\hline $\mathrm{TG}(\mathrm{mmol} / \mathrm{L})$ & $1.64 \pm 0.80$ & $1.75 \pm 1.105$ & 0.315 \\
\hline $\mathrm{TCHO}(\mathrm{mmol} / \mathrm{L})$ & $4.97 \pm 1.16$ & $4.55 \pm 0.97$ & $<0.001$ \\
\hline 8 am serum cortisol $(\mu \mathrm{g} / \mathrm{dL})$ & $16.92(12.32,23.99)$ & $12.35(9.71,16.87)$ & $<0.001$ \\
\hline $4 \mathrm{pm}$ serum cortisol $(\mu \mathrm{g} / \mathrm{dL})$ & $13.61(8.87,21.52)$ & $6.66(5.11,9.62)$ & $<0.001$ \\
\hline 0 am serum cortisol $(\mu \mathrm{g} / \mathrm{dL})$ & $11.67(6.33,19.35)$ & $2.67(1.77,4.50)$ & $<0.001$ \\
\hline 8 am $\mathrm{ACTH}(\mathrm{pg} / \mathrm{mL})$ & $2.00(0.99,7.74)$ & $18.54(12.30,26.62)$ & $<0.001$ \\
\hline $4 \mathrm{pm}$ ACTH (pg/mL) & $1.57(0.99,4.47)$ & $10.72(7.51,15.96)$ & $<0.001$ \\
\hline 0 am $\mathrm{ACTH}(\mathrm{pg} / \mathrm{mL})$ & $1.30(0.99,2.93)$ & $6.99(3.97,11.72)$ & $<0.001$ \\
\hline Urine cortisol $(\mu \mathrm{g} / 24 \mathrm{~h})$ & $963.3(568.1,1850.0)$ & $539.8(412.2,717.9)$ & $<0.001$ \\
\hline $\mathrm{CRP}(\mathrm{mg} / \mathrm{L})$ & $0.79(0.30,1.89)$ & $1.24(0.65,2.33)$ & 0.001 \\
\hline Longest diameter of adrenal nodule $(\mathrm{cm})$ & $2.70(2.20,3.60)$ & $1.60(1.00,2.30)$ & $<0.001$ \\
\hline
\end{tabular}

Results are presented as the mean \pm SD, median (interquartile range), or $n$ (\%). Abbreviations: CS: Cushing's syndrome; BMI: body mass index; SBP: systolic blood pressure; DBP: diastolic blood pressure; FPG: fasting plasma glucose; TG: triglyceride; TCHO: total cholesterol; ACTH: corticotropin; CRP: C-reactive protein.

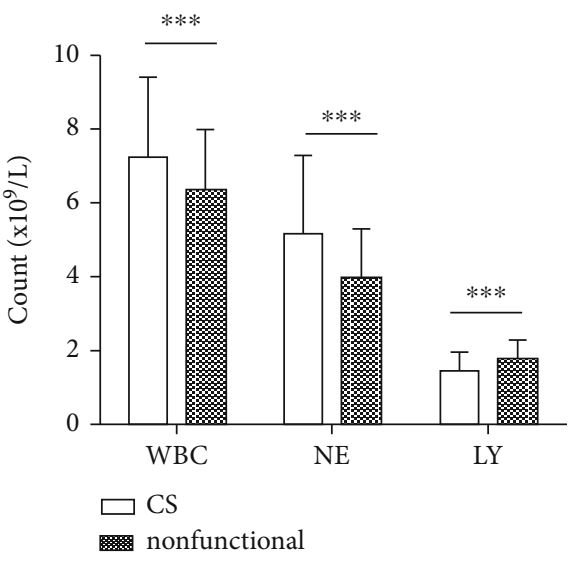

(a)

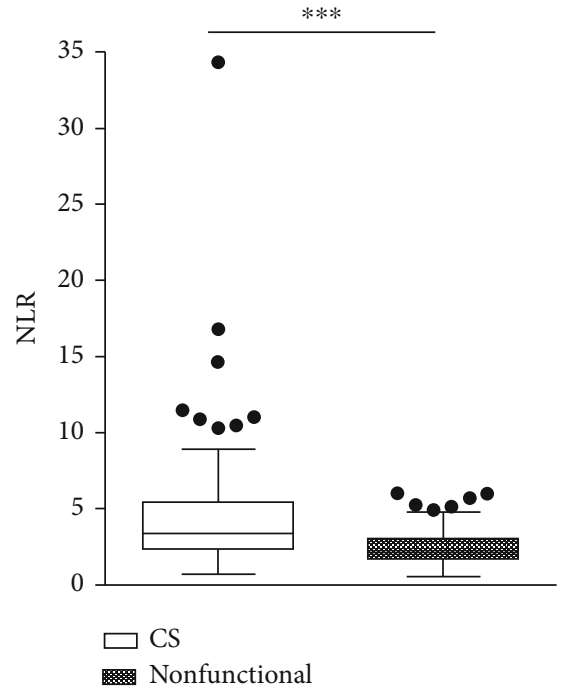

(b)

FIGURE 1: NLR and white blood cell, neutrophil, and lymphocyte counts in groups of patients with CS or a nonfunctional adrenal mass. (a) Neutrophil and lymphocyte counts in the two groups. The mean white blood cell counts $\left(7.25 \pm 2.16\right.$ vs. $\left.6.35 \pm 1.63\left(\times 10^{9} / \mathrm{L}\right), P<0.001\right)$ and neutrophil count $\left(5.17 \pm 2.11\right.$ vs. $\left.3.96 \pm 1.34\left(\times 10^{9} / \mathrm{L}\right), P<0.001\right)$ were significantly higher, and the mean lymphocyte count was significantly lower $\left(1.45 \pm 0.53\right.$ vs. $\left.1.75 \pm 0.53\left(\times 10^{9} / \mathrm{L}\right), P<0.001\right]$, in the CS group. Data are presented as the mean \pm SD. ${ }^{* * *} P<0.001$ (Student's $t$ test). (b) NLR for the two groups. The NLR $(3.38(2.33,5.45)$ vs. $2.13(1.74,3.00), P<0.001)$ was significantly higher in the CS group. Data are presented as the median (interquartile range). ${ }^{* * *} P<0.001$ (Mann-Whitney tests). The number in each group $=185$. Abbreviations: WBC: white blood cell count; NE: neutrophil count; LY: lymphocyte count; NLR: neutrophil-lymphocyte ratio; CS: Cushing's syndrome. 


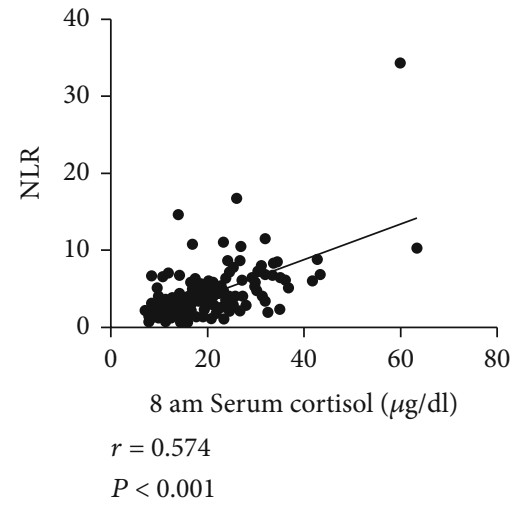

(a)

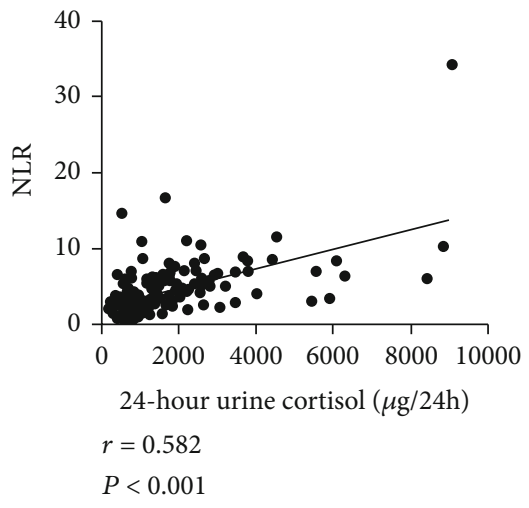

(b)

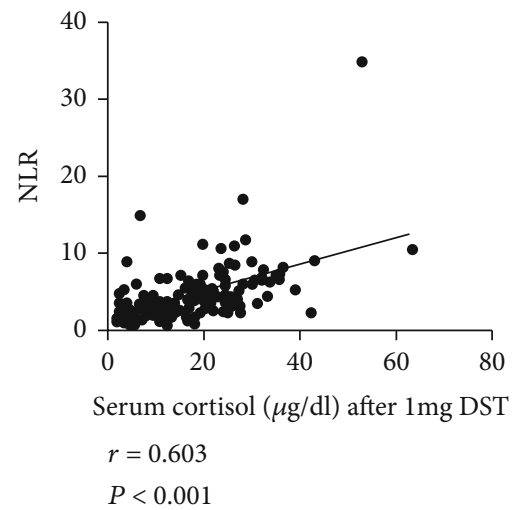

(c)

FIGURE 2: Scatter plots showing the correlation between the NLR and cortisol levels in the CS group. (a) NLR vs. 8 am serum cortisol concentration. (b). NLR vs. 24-hour urinary cortisol. (c) NLR vs. 8 am serum cortisol after $1 \mathrm{mg}$ DST. $R$ and $P$ values were determined by Spearman's correlation analysis. $N=185$. Abbreviations: NLR: neutrophil-lymphocyte ratio; CS: Cushing's syndrome; DST: dexamethasone suppression test.

TABLE 2: Comparison of hormone concentrations, NLR, and PLR in patients with overt CS and AIMCE.

\begin{tabular}{lccc}
\hline & Overt CS $(n=141)$ & AIMCE $(n=44)$ & $P$ \\
\hline 8 am serum cortisol $(\mu \mathrm{g} / \mathrm{dL})$ & $19.76(13.79,25.45)$ & $13.82(10.51,16.06)$ & $<0.001$ \\
8 am ACTH $(\mathrm{pg} / \mathrm{mL})$ & $1.23(0.90,4.32)$ & $8.43(4.30,11.85)$ & $<0.001$ \\
NE $\left(\times 10^{9} / \mathrm{L}\right)$ & $5.58 \pm 2.16$ & $3.85 \pm 1.22$ & $<0.001$ \\
LY $\left(\times 10^{9} / \mathrm{L}\right)$ & $1.35 \pm 0.47$ & $1.77 \pm 0.58$ & $<0.001$ \\
NLR & $4.00(2.70,6.18)$ & $2.16(1.60,3.07)$ & $<0.001$ \\
\hline
\end{tabular}

Results are presented as the mean \pm SD or as the median (interquartile range). Abbreviations: ACTH: corticotropin; NE: neutrophil count; LY: lymphocyte count; NLR: neutrophil-lymphocyte ratio; CS: Cushing's syndrome; AIMCE: adrenal incidentaloma with mild autonomous cortisol excess.

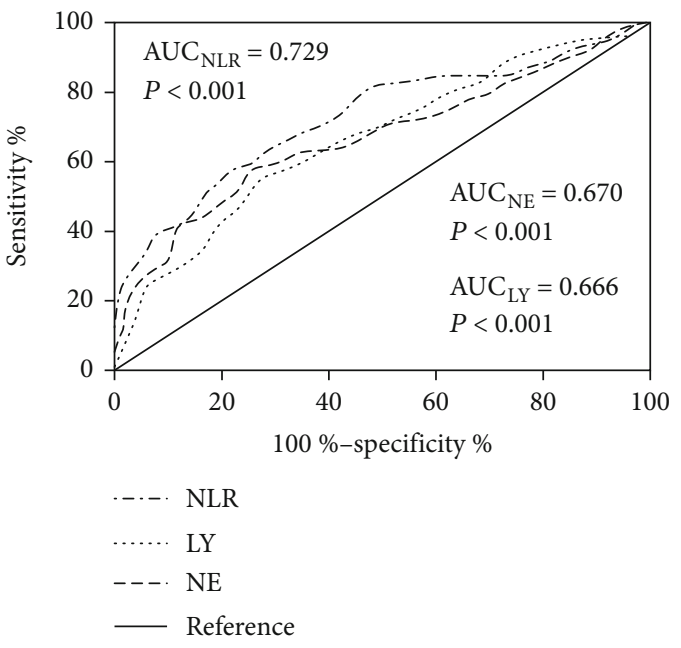

FIGURE 3: Receiver operating characteristic curves of the NLR, NE, and LY for diagnosis of CS. $N=370$. Abbreviations: NLR: neutrophil-lymphocyte ratio; NE: neutrophil count; LY: lymphocyte count; AUC: area under the curve; CS: Cushing's syndrome.

patients with CS was significantly higher than that in individuals with a nonfunctional adrenal mass. Conversely, the CRP concentration was lower in CS patients than in the nonfunctional adrenal mass group, indicating that differ- ences in the NLR between these two groups do not reflect inflammatory status.

We found that the CS group was significantly younger than the nonfunctional group. A previous study shows that the NLR is positively associated with age; older people have a relatively high NLR [29]. This suggests that the higher NLR in the CS group was not due to age differences. Because patients with CS show varied clinical complications [30], an adrenal mass might be detected earlier than in patients with asymptomatic nonfunctional adenoma. In our study, we excluded those with adrenal lymphoma and adrenal metastatic carcinoma. In these cases, the NLR does not reflect cortisol levels; this is because adrenal malignant masses might affect inflammatory parameters, including the NLR.

Current screening tests for CS include late-night saliva/serum cortisol concentration, 24-hour UFC, and DSTs [20]. Although these tests have high diagnostic accuracy and are feasible, their widespread use is limited by stringent requirements with respect to specimen collection and patient compliance, as well as by the need for specialized equipment, which is a problem in underdeveloped countries and small hospitals. By contrast, the NLR can be calculated easily from routine blood test data. Compared with traditional initial tests for CS diagnosis, the NLR has several advantages. In the case of the 24-hour UFC test, it is not easy for patients to provide a complete set of 24-hour urine samples of the 
TABLE 3: Comparison of different initial screen markers used to diagnose CS.

\begin{tabular}{lccc}
\hline & Sensitivity & Specificity & Weighted Youden index $^{\triangle}$ \\
\hline 0 am serum cortisol $(\mu \mathrm{g} / \mathrm{dL})$ & $98.8 \%^{* * *}$ & $26.6 \%^{* * *}$ & 0.543 \\
24-hour urine free cortisol & $67.1 \%^{* *}$ & $70.5 \%^{* *}$ & 0.362 \\
Serum cortisol after 1 mg DST $(\mu \mathrm{g} / \mathrm{dL})$ & $100 \%^{* * *}$ & $89.1 \%^{* * *}$ & 0.935 \\
NLR & $80.0 \%$ & $54.1 \%$ & 0.445 \\
\hline
\end{tabular}

Abbreviations: DST: dexamethasone suppression test; NLR: neutrophil-lymphocyte ratio; CS: Cushing's syndrome. ${ }^{* *} P<0.01$ and ${ }^{* * *} P<0.001, v s$. NLR.

${ }^{\triangle}$ weighted coefficient of sensitivity $\omega=0.7$.

appropriate total volume. Moreover, excessive fluid intake and other physiological or pathological conditions can affect UFC test results [31]. A falsely low UFC can occur when the creatinine clearance rate falls below $60 \mathrm{~mL} / \mathrm{min}$ [32]. In the case of the late-night saliva/serum cortisol test, samples have to be collected late at night, which is inconvenient. Also, it cannot be used for shift workers or for those with variable bedtimes [20]. Patients who smoke cigarettes have higher salivary cortisol concentrations than nonsmokers [33]. Pain and stress caused by blood drawing can affect serum cortisol levels. Studies show that the sensitivity and specificity of UFC for CS diagnosis are 65-97\% and 56-100\%, respectively, due to the use of different cut-off values and methods [34, 35]. The sensitivity and specificity of the late-night serum cortisol test with a cut-off of $1.8 \mu \mathrm{g} / \mathrm{dL}$ are $100 \%$ and $20.2 \%$, respectively [36], whereas those of postdexamethasone serum cortisol tests to less than $1.8 \mu \mathrm{g} / \mathrm{dL}$ are greater than $95 \%$ and $80 \%$, respectively [37]. The weighted Youden index suggests that the diagnostic accuracy of the NLR in our study is similar to that of the late-night serum cortisol concentration and 24-hour UFC tests.

Thus, we propose that NLR may be an initial screening test for patients with an adrenal mass that lack factors that could affect neutrophil or lymphocyte counts. Calculating the NLR is easy and can be done in primary care clinics and healthcare facilities in underdeveloped regions. For attention, although WBC counts, neutrophil counts, and the NLR were significantly higher in the CS group than in the nonfunctional group, WBC counts and neutrophil counts are still in the normal range.

Taken together, the results presented herein indicate that the NLR is an easily measurable, reproducible, and inexpensive marker for initial screening of CS in patients with an adrenal mass. Undoubtedly, all patients with an adrenal mass require evaluation in an endocrinological unit. A complete adrenal function evaluation, including cortisol, aldosterone, and catecholamine levels, is required. Also, a $1 \mathrm{mg}$ overnight DST is the most important parameter for diagnosis under the current guidelines, and this can be done easily in endocrinological units. The NLR test, which can be performed in nonspecialist units, could also provide useful information when no endocrinologist is available.

The present study has several limitations. First, it was performed at a single center in China and included a relatively small number of patients. Second, the retrospective design means that the NLR could only be measured preoperatively. It is unclear whether the NLR in patients with CS falls after adrenalectomy, or whether changes in the NLR predict CS outcome. Further studies are needed to confirm our results.

\section{Conclusions}

The NLR is easier to calculate during routine blood testing than current initial tests for CS. We found that the NLR in patients with CS was significantly higher than that in patients with a nonfunctional adrenal mass. A high NLR correlated positively with cortisol levels. A NLR $\geq 2.2$ may be used as a cut-off for screening tests for initial diagnosis of CS in patients with an adrenal mass.

\section{Data Availability}

The datasets used and analyzed during the current study are available from the corresponding author on reasonable request.

\section{Conflicts of Interest}

The authors declare no conflicts of interest regarding publication of this paper.

\section{Authors' Contributions}

W.W., Y.G., and J.Z. conceived and designed the study. W.W., J.W., and Y.G. drafted and revised the manuscript. W.W., J.W., C.S., and S.Z. collected and analyzed the data. Wei Wang and Jianing Wang contributed equally to this work.

\section{Acknowledgments}

This work was supported by the Youth Clinical Research Project of Peking University First Hospital (Grant No. 2019CR22 to WW).

\section{References}

[1] L. Barzon, N. Sonino, F. Fallo, G. Palu, and M. Boscaro, "Prevalence and natural history of adrenal incidentalomas," European Journal of Endocrinology, vol. 149, no. 4, pp. 273-285, 2003.

[2] R. T. Kloos, M. D. Gross, I. R. Francis, M. Korobkin, and B. Shapiro, "Incidentally discovered adrenal masses," Endocrine Reviews, vol. 16, no. 4, pp. 460-484, 1995.

[3] G. Mansmann, J. Lau, E. Balk, M. Rothberg, Y. Miyachi, and S. R. Bornstein, "The clinically inapparent adrenal mass: update in diagnosis and management," Endocrine Reviews, vol. 25, no. 2, pp. 309-340, 2004.

[4] F. Mantero, M. Terzolo, G. Arnaldi et al., "A survey on adrenal incidentaloma in Italy. Study Group on Adrenal Tumors of the Italian Society of Endocrinology," The Journal of Clinical 
Endocrinology and Metabolism, vol. 85, no. 2, pp. 637-644, 2000.

[5] S. Bovio, A. Cataldi, G. Reimondo et al., "Prevalence of adrenal incidentaloma in a contemporary computerized tomography series," Journal of Endocrinological Investigation, vol. 29, no. 4, pp. 298-302, 2006.

[6] M. Fassnacht, W. Arlt, I. Bancos et al., "Management of adrenal incidentalomas: European Society of Endocrinology Clinical Practice Guideline in collaboration with the European Network for the Study of Adrenal Tumors," European Journal of Endocrinology, vol. 175, no. 2, pp. G1-G34, 2016.

[7] J. Lindholm, S. Juul, J. O. L. Jørgensen et al., "Incidence and late prognosis of Cushing's syndrome: a population-based study," The Journal of Clinical Endocrinology and Metabolism, vol. 86, no. 1, pp. 117-123, 2001.

[8] B. Swearingen, B. M. Biller, Barker FG 2nd et al., "Long-term mortality after transsphenoidal surgery for Cushing disease," Annals of Internal Medicine, vol. 130, no. 10, pp. 821-824, 1999.

[9] O. M. Dekkers, E. Horváth-Puhó, J. O. L. Jørgensen et al., "Multisystem morbidity and mortality in Cushing's syndrome: a cohort study," The Journal of Clinical Endocrinology and Metabolism, vol. 98, no. 6, pp. 2277-2284, 2013.

[10] M. J. Bolland, I. M. Holdaway, J. E. Berkeley et al., "Mortality and morbidity in Cushing's syndrome in New Zealand," Clinical Endocrinology, vol. 75, no. 4, pp. 436-442, 2011.

[11] G. D. Hammer, J. B. Tyrrell, K. R. Lamborn et al., "Transsphenoidal microsurgery for Cushing's disease: initial outcome and long-term results," The Journal of Clinical Endocrinology and Metabolism, vol. 89, no. 12, pp. 6348-6357, 2004.

[12] Chinese Society of Endocrinology, "Experts consensus for Cushing's syndrome (2011)," Chinese Journal of Endocrinology and Metabolism, vol. 28, pp. 96-102, 2012.

[13] H. Masri-Iraqi, E. Robenshtok, G. Tzvetov, Y. Manistersky, and I. Shimon, "Elevated white blood cell counts in Cushing's disease: association with hypercortisolism," Pituitary, vol. 17, no. 5, pp. 436-440, 2014.

[14] C. Tatsi, R. Boden, N. Sinaii et al., "Decreased lymphocytes and increased risk for infection are common in endogenous pediatric Cushing syndrome," Pediatric Research, vol. 83, no. 2, pp. 431-437, 2018.

[15] Y. F. He, H. Q. Luo, W. Wang et al., "Preoperative NLR and PLR in the middle or lower ESCC patients with radical operation," European Journal of Cancer Care, vol. 26, no. 2, 2017.

[16] A. Passardi, E. Scarpi, L. Cavanna et al., "Inflammatory indexes as predictors of prognosis and bevacizumab efficacy in patients with metastatic colorectal cancer," Oncotarget, vol. 7, no. 22, pp. 33210-33219, 2016.

[17] B. Qin, N. Ma, Q. Tang et al., "Neutrophil to lymphocyte ratio (NLR) and platelet to lymphocyte ratio (PLR) were useful markers in assessment of inflammatory response and disease activity in SLE patients," Modern Rheumatology, vol. 26, no. 3, pp. 372-376, 2016.

[18] N. Xu, X. F. Tang, J. J. Xu et al., "Predictive value of neutrophil to lymphocyte ratio on long-term outcomes of acute myocardial infarction patients with multivessel disease," Chinese Journal of Cardiology, vol. 47, no. 1, pp. 42-48, 2019.

[19] J. F. Condado, P. Junpaparp, J. N. Binongo et al., "Neutrophillymphocyte ratio (NLR) and platelet-lymphocyte ratio (PLR) can risk stratify patients in transcatheter aortic-valve replace- ment (TAVR)," International Journal of Cardiology, vol. 223, pp. 444-449, 2016.

[20] L. K. Nieman, B. M. Biller, J. W. Findling et al., "The diagnosis of Cushing's syndrome: an Endocrine Society clinical practice guideline," The Journal of Clinical Endocrinology and Metabolism, vol. 93, no. 5, pp. 1526-1540, 2008.

[21] J. W. Funder, R. M. Carey, F. Mantero et al., "The management of primary aldosteronism: case detection, diagnosis, and treatment: an Endocrine Society clinical practice guideline," The Journal of Clinical Endocrinology and Metabolism, vol. 101, no. 5, pp. 1889-1916, 2016.

[22] J. W. Lenders, Q. Y. Duh, G. Eisenhofer et al., "Pheochromocytoma and paraganglioma: an Endocrine Society clinical practice guideline," The Journal of Clinical Endocrinology and Metabolism, vol. 99, no. 6, pp. 1915-1942, 2014.

[23] M. Reincke, "Subclinical Cushing's syndrome," Endocrinology and Metabolism Clinics of North America, vol. 29, no. 1, pp. 43-56, 2000.

[24] M. Terzolo, A. Pia, and G. Reimondo, "Subclinical Cushing's syndrome: definition and management," Clinical Endocrinology, vol. 76, no. 1, pp. 12-18, 2012.

[25] J. M. Davis, J. D. Albert, K. J. Tracy et al., "Increased neutrophil mobilization and decreased chemotaxis during cortisol and epinephrine infusions," The Journal of Trauma, vol. 31, no. 6, pp. 725-732, 1991.

[26] W. C. Liles, D. C. Dale, and S. J. Klebanoff, "Glucocorticoids inhibit apoptosis of human neutrophils," Blood, vol. 86, no. 8, pp. 3181-3188, 1995.

[27] M. Nakagawa, T. Terashima, Y. D’yachkova, G. P. Bondy, J. C. Hogg, and S. F. van Eeden, "Glucocorticoid-induced Granulocytosis,” Circulation, vol. 98, no. 21, pp. 2307-2313, 1998.

[28] T. Dong, L. Zhi, B. Bhayana, and M. X. Wu, "Cortisol-induced immune suppression by a blockade of lymphocyte egress in traumatic brain injury," Journal of Neuroinflammation, vol. 13, no. 1, p. 197, 2016.

[29] J. Li, Q. Chen, X. Luo et al., "Neutrophil-to-lymphocyte ratio positively correlates to age in healthy population," Journal of Clinical Laboratory Analysis, vol. 29, no. 6, pp. 437-443, 2015.

[30] R. Pivonello, A. M. Isidori, M. C. de Martino, J. Newell-Price, B. M. K. Biller, and A. Colao, "Complications of Cushing's syndrome: state of the art," The Lancet Diabetes and Endocrinology, vol. 4, no. 7, pp. 611-629, 2016.

[31] M. V. Mericq and G. B. Cutler Jr., "High fluid intake increases urine free cortisol excretion in normal subjects," The Journal of Clinical Endocrinology and Metabolism, vol. 83, no. 2, pp. 682684, 1998.

[32] K. C. Chan, L. C. Lit, E. L. Law et al., "Diminished urinary free cortisol excretion in patients with moderate and severe renal impairment," Clinical Chemistry, vol. 50, no. 4, pp. 757-759, 2004.

[33] E. Badrick, C. Kirschbaum, and M. Kumari, "The relationship between smoking status and cortisol secretion," The Journal of Clinical Endocrinology and Metabolism, vol. 92, no. 3, pp. 819824, 2007.

[34] F. Ceccato, G. Marcelli, M. Martino et al., "The diagnostic accuracy of increased late night salivary cortisol for Cushing's syndrome: a real-life prospective study," Journal of Endocrinological Investigation, vol. 42, no. 3, pp. 327-335, 2019.

[35] A. Oßwald, R. Wang, F. Beuschlein et al., "Performance of LC-MS/MS and immunoassay based 24-h urine free 
cortisol in the diagnosis of Cushing's syndrome," The Journal of Steroid Biochemistry and Molecular Biology, vol. 190, pp. 193-197, 2019.

[36] F. Pecori Giraldi, A. G. Ambrogio, M. de Martin, L. M. Fatti, M. Scacchi, and F. Cavagnini, "Specificity of first-line tests for the diagnosis of Cushing's syndrome: assessment in a large series," The Journal of Clinical Endocrinology and Metabolism, vol. 92, no. 11, pp. 4123-4129, 2007.

[37] P. J. Wood, J. H. Barth, D. B. Freedman, L. Perry, and B. Sheridan, "Evidence for the low dose dexamethasone suppression test to screen for Cushing's syndrome-recommendations for a protocol for biochemistry laboratories," Annals of Clinical Biochemistry, vol. 34, no. 3, pp. 222-229, 1997. 